\title{
ON ROTATION-AUTOMORPHIC FUNCTIONS
}

\author{
RAUNO AULASKARI and TUOMAS SORVALI
}

1.

In this paper we shall consider classes of analytic or meromorphic functions of a complex variable which are generalizations of classical automorphic functions, the main interest being in the normality of the functions.

Let $(X, d)$ and $(Y, \varrho)$ be metric spaces. Consider a family $\mathscr{F}$ of continuous functions $f: X \rightarrow Y$. The family $\mathscr{F}$ is normal if every sequence $\left(f_{n}\right) \subset \mathscr{F}$ contains a subsequence $\left(f_{k}\right)$ which converges uniformly on every compact subset of $X$.

Let $\Omega$ be a group of isometric automorphisms of $X$.

Definition 1. A function $f: X \rightarrow Y$ is normal (with respect to $\Omega$ ) if $\{f \circ T \mid T \in \Omega\}$ is a normal family.

If $f$ is a meromorphic function, then the normality of $f$ is closely related to the following property of $f$.

Definition 2. A function $f: X \rightarrow Y$ is of bounded stretching if there exists an $M>0$ such that

$$
\limsup _{x^{\prime} \rightarrow x} \frac{\varrho\left(f(x), f\left(x^{\prime}\right)\right)}{d\left(x, x^{\prime}\right)} \leqq M
$$

for all $x \in X$.

Let $\Omega^{\prime}$ be the group of the isometric automorphisms of $Y$.

Definition 3. A function $f: X \rightarrow Y$ is homomorphism-automorphic with respect to a subgroup $\Gamma \subset \Omega$ if for every $T \in \Gamma$ there exists a $j(T) \in \Omega^{\prime}$ such that

$$
f \circ T=j(T) \circ f .
$$

Remark 1. Let $f$ be a homomorphism-automorphic function and let $F$ be a fundamental set of $\Gamma$. Then $f$ is of bounded stretching if and only if there exists an $M>0$ such that (1.1) holds for all $x \in F$.

Received May 19, 1980. 
2.

Let $X$ be the unit disk $D$ and let $d$ be the non-euclidean metric of $D$. For the space $(Y, \varrho)$ we choose the Rieman sphere $\hat{C}$ with the spherical metric $\varrho$. Then, by (1.1), a meromorphic function $f: D \rightarrow \hat{C}$ is of bounded stretching if and only if

$$
\sup _{z \in D}\left(1-|z|^{2}\right) \frac{\left|f^{\prime}(z)\right|}{1+|f(z)|^{2}} \leqq M
$$

for some $M>0$.

Let $\Omega$ be the group of the Möbius transformations $T: D \rightarrow D$. Then we have ([3]):

TheOREM 1. A meromorphic function $f: D \rightarrow \hat{C}$ is normal in $D$ if and only if (2.1) holds, i.e., $f$ is of bounded stretching.

3.

Suppose that $\Gamma$ is a Fuchsian group acting on $D$, and let $f$ be a non-constant meromorphic function in $D$. Suppose that $f$ is homomorphism-automorphic with respect to $\Gamma$. Since $f$ is meromorphic and $T$ is conformal, it follows from (1.2) that $j(T)$ is sense-preserving, i.e., $j(T)$ is a rotation. On the other hand, if $T$ is given, then (1.2) defines $j(T)$ uniquely. It follows that $\Gamma^{\prime}=\{j(T) \mid T \in \Gamma\}$ is a group of rotations of the sphere and $j: \Gamma \rightarrow \Gamma^{\prime}$ is a homomorphism. In this case we call $f$ a rotation-automorphic function. The following special cases are well-known:

(1) If $\Gamma^{\prime}$ contains only rotations $w \mapsto e^{i \varphi} w$, then $f$ is a characterautomorphic function (cf. [4]).

(2) If $\Gamma^{\prime}$ is trivial, then $f$ is an automorphic function.

Combining Remark 1 and Theorem 1 we obtain

THEOREM 2. Let $f: D \rightarrow \hat{C}$ be a rotation-automorphic function with respect to a Fuchsian group $\Gamma$, and let $F$ be a fundamental set of $\Gamma$. Then $f$ is normal if and only if

$$
\sup _{z \in F}\left(1-|z|^{2}\right) \frac{\left|f^{\prime}(z)\right|}{1+|f(z)|^{2}} \leqq M
$$

holds for some $M>0$.

4.

Let $(Y, \varrho)$ be the finite plane $C$ with the euclidean metric, and let $f: D \rightarrow C$ be a non-constant analytic function. Then $f$ is of bounded stretching if and only if 


$$
\sup _{z \in D}\left(1-|z|^{2}\right)\left|f^{\prime}(z)\right| \leqq M
$$

for some $M>0$, i.e., if and only if $f$ is a Bloch function.

A Bloch function $f$ need not be normal in the sense of Definition 1. However, every sequence $f \circ T_{n}, T_{n} \in \Omega$, contains a subsequence which converges either to an analytic function or to $\infty$, uniformly on compact subsets of $D$. Hence a Bloch function is normal if the euclidean metric of $C$ is replaced by the spherical metric.

Let $f: D \rightarrow C$ be a homomorphism-automorphic function with respect to a Fuchsian group $\Gamma$. Then $\Gamma^{\prime}$ consists of translations $w \mapsto w+\omega$, and $f$ is called an additive automorphic function (cf. [2]). Especially, if $\Gamma^{\prime}$ is trivial then $f$ is automorphic.

Let $F$ be a fundamental set of $\Gamma$. By Remark 1, an additive automorphic function $f$ is a Bloch function if and only if

$$
\sup _{z \in F}\left(1-|z|^{2}\right)\left|f^{\prime}(z)\right| \leqq M
$$

holds for some $M>0$.

REMARK 2 . Let $(X, d)$ and $(Y, \varrho)$ be unit disks with the non-euclidean metric $d$ and let $f: D \rightarrow D$ be an analytic homomorphism-automorphic function with respect to a Fuchsian group $\Gamma$. If $\Gamma^{\prime}$ is a Fuchsian group, then $f$ induces an analytic function from $D / \Gamma$ into $D / \Gamma^{\prime}$. In this sense, analytic functions between Riemann surfaces are special cases of homomorphism-automorphic functions.

5.

In this section we consider the existence of homomorphism-, rotation-, and additive automorphic functions.

Let $X$ and $Y$ be Riemann surfaces and $f: X \rightarrow Y$ a non-constant analytic function. Suppose that the covering surface $(X, f)$ of $Y$ is universal, let $\Gamma_{0}$ be the cover transformation group and denote by $H^{\prime}$ the group of all conformal selfmappings of $Y$. A conformal mapping $T: X \rightarrow X$ is a lifting of $S \in H^{\prime}$ if $f \circ T=S$ $\circ f$. It follows that every $S \in H^{\prime}$ has liftings. Especially, all cover transformations are obtained as liftings of the identity mapping of $Y$.

Let $H$ be the set of all liftings of the mappings of $H^{\prime}$. Then $H$ is a group and $j: H \rightarrow H^{\prime}, j(T)=S$, is a homomorphism whose kernel is the cover transformation group $\Gamma_{0}$. This can be stated differently also as follows:

THEOREM 3. The mapping $f: X \rightarrow Y$ is automorphic with respect to $\Gamma_{0}$ and homomorphism-automorphic with respect to $H$. The group $H$ contains as a subgroup every group $\Gamma$ with respect to which $f$ is homomorphism-automorphic. 
If $\Gamma^{\prime}$ is a subgroup of $H^{\prime}$, then we can similarly as above define $\Gamma$ as the group of all liftings of the mappings of $\Gamma^{\prime}$. Then $\Gamma \subset H$ and $f$ is homomorphismautomorphic with respect to $\Gamma$. Suppose that $\Gamma^{\prime}$ is properly discontinuous in $Y$. Then the same holds true of $\Gamma$ in $X$. In this case $\Gamma$ can also be characterized as a cover transformation group (cf. $[1, \mathrm{II} .4 \mathrm{C}]$ ):

THEOREM 4. $\Gamma$ is the cover transformation group of $(X, \varphi \circ f)$ over $Z=Y / \Gamma^{\prime}$ where $\varphi: Y \rightarrow Z$ is the canonical projection.

Proof. Evidently $\Gamma$ is a subgroup of the cover transformation group in question. From the construction of $Z$ it follows that whenever $x_{1}$ and $x_{2}$ are two points of $X$ lying over the same point of $Z$, there is an $S \in \Gamma$ for which $S\left(x_{1}\right)=x_{2}$. From this it follows that $\Gamma$ contains all cover transformations of $(X, \varphi \circ f)$.

Note that $(X, \varphi \circ f)$ is a universal covering surface of $Z$ only if $\Gamma^{\prime}$ is in addition also fixed point free in $Y$.

Suppose now that $Y$ is a domain in $\hat{C}$ having the unit disk $D$ as a universal covering surface. If $\Gamma^{\prime}$ is a properly discontinuous group of conformal selfmappings of $Y$, then $\Gamma$ is a Fuchsian group and the projection $f: D \rightarrow Y$ is homomorphism-automorphic with respect to $\Gamma$.

In order to construct a rotation-automorphic function $f: D \rightarrow \hat{C}$, choose $Y$ such that there exists a properly discontinuous group $\Gamma^{\prime}$ of rotations of the sphere mapping $Y$ onto itself. This can be done e.g. by choosing for the group $\Gamma^{\prime}$ a group of a regular solid and letting $Y$ be the sphere $\hat{C}$ punctured at the fixed points of $\Gamma^{\prime}$.

To obtain an additive automorphic function $f: D \rightarrow \mathrm{C}$, let $\Gamma^{\prime}$ be the group generated by the translations $z \rightarrow z+\omega_{1}$ and $z \rightarrow z+\omega_{2}, \operatorname{Im}\left(\omega_{1} / \omega_{2}\right)>0$, and let $Y$ be the plane $C$ punctured at the points $m \omega_{1}+n \omega_{2}, m$ and $n$ integers.

6.

Next we give an explicit example of a rotation-automorphic function such that $\Gamma^{\prime}$ is not fixed point free in $Y$.

Let $\Gamma$ be the group generated by the parabolic transformations

$$
T_{1}(z)=\frac{(1-i) z+1}{z+1+i}, \quad T_{2}(z)=\frac{(1+i) z+1}{z+1-i} .
$$

Then $\Gamma$ has a fundamental polygon $F$ whose sides lie on the circles 


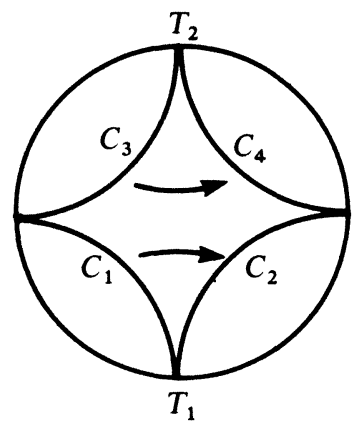

$$
\begin{aligned}
& C_{1}=\{z|| z-(-1-i) \mid=1\} \\
& C_{2}=\{z|| z-(1-i) \mid=1\} \\
& C_{3}=\{z|| z-(-1+i) \mid=1\} \\
& C_{4}=\{z|| z-(1+i) \mid=1\} .
\end{aligned}
$$

Figure 1.

The transformation $T_{1}$ sends $C_{1}$ onto $C_{2}$ and $T_{2}$ sends $C_{3}$ onto $C_{4}$ (see Figure 1.).

Let $S_{2}$ be the rotation

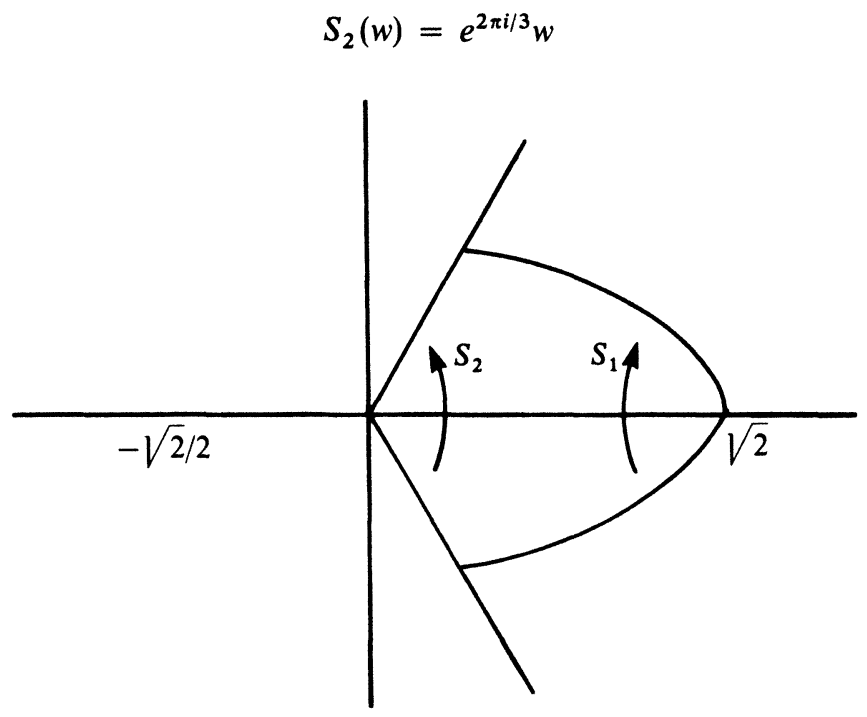

Figure 2.

and $S_{1}$ the rotation whose fixed points are $-\sqrt{2} / 2$ and $\sqrt{2}$ and multiplier equals $e^{2 \pi i / 3}$. Let $\Gamma^{\prime}$ be the group generated by $S_{1}$ and $S_{2}$. Define a homomorphism $j: \Gamma \rightarrow \Gamma^{\prime}$ by $j\left(T_{i}\right)=S_{i}, i=1,2$. Let $F^{\prime}$ be the domain bounded by the rays $w=t e^{ \pm \pi i / 3}, t \geqq 0$, and by the circular arcs through the fixed points $\sqrt{2}$ and $-\sqrt{2} / 2$ (Figure 2). Consider $F$ and $F^{\prime}$ as quadrilaterals. By symmetry, both have the modulus one. Hence there is a conformal mapping $f$ from $F$ onto 
$F^{\prime}$ which sends vertices onto vertices and equivalent sides onto equivalent sides, respectively. The function $f$ can be continued to $D$ by the formula (1.2). Then $f$ is analytic in $D$. Moreover, $f$ is rotation-automorphic with respect to $\Gamma$. Since $\Gamma^{\prime}$ is a subgroup of the cubic group, it is properly discontinuous in $\hat{C}$. Moreover, the transformations of $\Gamma^{\prime}$ keep

$$
Y=\hat{\mathrm{C}} \backslash\left\{0, \sqrt{2},-\sqrt{2} / 2, \sqrt{2} e^{2 \pi i / 3}, \sqrt{2} e^{-2 \pi i / 3},(-\sqrt{2} / 2) e^{2 \pi i / 3},(-\sqrt{2} / 2) e^{-2 \pi i / 3}, \infty\right\}
$$

invariant. Since $S_{1}\left(S_{2}^{-1}\left(S_{1}\left(F^{\prime}\right)\right)\right)=F^{\prime}$, the rotation $S_{1} \circ S_{2}^{-1} \circ S_{1} \neq i d$ has a fixed point in $F^{\prime}$. Hence $\Gamma^{\prime}$ is not fixed point free in $Y$.

Since $f$ omits eight values, $f$ is normal in $D$.

7.

Let $f$ be a rotation-automorphic function with respect to $\Gamma$. We consider conditions under which $f$ is normal in $D$.

Let $F$ be a normal fundamental polygon of $\Gamma$.

Definition 4. (cf. [2]) The fundamental domain $F$ is thick if for each sequence $z_{n} \in F, n=1,2, \ldots$, there is a sequence $z_{n}^{\prime}$ and constants $r>0, r^{\prime}>0$ such that $d\left(z_{n}, z_{n}^{\prime}\right)<r$ and

$$
U\left(z_{n}^{\prime}, r^{\prime}\right)=\left\{z \mid d\left(z_{n}^{\prime}, z\right)<r^{\prime}\right\} \subset F .
$$

Lemma. Let $z_{n} \in F$ such that $\left|z_{n}\right| \rightarrow 1$ as $n \rightarrow \infty$. If $r>0$ and $0<R<1$, denote $U_{n}=U\left(z_{n}, r\right)$ and $D_{R}=\{z|| z \mid<R\}$. Then $T\left(U_{n}\right) \cap D_{R} \neq \varnothing$ for finitely many $T \in \Gamma$ and $n \in \mathrm{N}$ only.

Proof. Choose $\left.R^{\prime} \in\right] R, 1\left[\right.$ such that $U(z, r) \cap D_{R}=\varnothing$ if $|z| \geqq R^{\prime}$. Then $T(\bar{F}) \cap D_{R^{\prime}} \neq \varnothing$ for finitely many transformations $T \in \Gamma$ only. On the other hand, for every $T \in \Gamma$ the set $\left\{T\left(z_{n}\right) \mid n=1,2, \ldots\right\} \cap D_{R^{\prime}}$ is finite. Combining these trivial observations we infer that $\left|T\left(z_{n}\right)\right|<R^{\prime}$ for at most finitely many $T \in \Gamma$ and $n \in \mathbb{N}$.

THEOREM 5. If $F$ is thick and

$$
\iint_{F}\left(\frac{\left|f^{\prime}(z)\right|}{1+|f(z)|^{2}}\right)^{2} d \sigma_{z}<\infty
$$

then $f$ is normal in $D$.

Proof. Suppose, on the contrary, that $f$ is not normal in $D$. Then (3.1) does not hold. Thus there is a sequence $z_{n} \in F$ such that $\left|z_{n}\right| \rightarrow 1$ and 


$$
\left(1-\left|z_{n}\right|^{2}\right) \frac{\left|f^{\prime}\left(z_{n}\right)\right|}{1+\left|f\left(z_{n}\right)\right|^{2}} \rightarrow \infty
$$

as $n \rightarrow \infty$.

Choose $r>0$. By the above lemma, the thickness of $F$ and (7.1), we have

$$
\lim _{n \rightarrow \infty} \iint_{U\left(z_{n}, r\right)}\left(\frac{\left|f^{\prime}(z)\right|}{1+|f(z)|^{2}}\right)^{2} d \sigma_{z}=0 .
$$

We can select a subsequence, also denoted by $z_{n}$, such that

$$
\sum_{n=1}^{\infty} \iint_{U\left(z_{n}, r\right)}\left(\frac{\left|f^{\prime}(z)\right|}{1+|f(z)|^{2}}\right)^{2} d \sigma_{z}<\pi
$$

Let

$$
g_{n}(\zeta)=f\left(\frac{\zeta+z_{n}}{1+\bar{z}_{n} \zeta}\right)
$$

Then

$$
\iint_{U(0, r)}\left(\frac{\left|g_{n}^{\prime}(\zeta)\right|}{1+\left|g_{n}(\zeta)\right|^{2}}\right)^{2} d \sigma_{\zeta}=\iint_{U\left(z_{n}, r\right)}\left(\frac{\left|f^{\prime}(z)\right|}{1+|f(z)|^{2}}\right)^{2} d \sigma_{z}
$$

is equal to the spherical area of the surface onto which $g_{n}$ maps the disk $U(0, r)$. It follows from (7.4) and (7.5) that the family $\left\{g_{n}\right\}$ omits in $U(0, r)$ a set of positive spherical area. Hence the family $\left\{g_{n}\right\}$ is normal in $U(0, r)$. Therefore

$$
\frac{\left|g_{n}^{\prime}(0)\right|}{1+\left|g_{n}(0)\right|^{2}}=\left(1-\left|z_{n}\right|^{2}\right) \frac{\left|f^{\prime}\left(z_{n}\right)\right|}{1+\left|f\left(z_{n}\right)\right|^{2}} \leqq M<\infty
$$

for $n=1,2, \ldots$ This contradicts (7.2).

\section{REFERENCES}

1. L. V. Ahlfors and L. Sario, Riemann surfaces, (Princeton Mathematical Series 26) Princeton University Press, Princeton, N.J., 1960.

2. R. Aulaskari, On normal additive automorphic functions, Ann. Acad. Sci. Fenn. Ser.A I Math. Dissertationes 23 (1978), 1-53.

3. O. Lehto and K. I. Virtanen, Boundary behaviour and normal meromorphic functions, Acta Math. 97 (1957), 47-65.

4. Ch. Pommerenke, On normal and automorphic functions, Michigan Math. J. 21 (1974), 193-202.

JOENSUUN KORKEAKOULU

PL 111

80101 JOENSUU 10

FINLAND 